\title{
Effect of Different Levels of Nitrogen and Phosphorus on Herbage Yield of Palmarosa (Cymbopogan martini var. Motia)
}

\author{
M Jayalakshmi and S G Wankhade \\ Depertment of Medicinal and Aromatic Plants, \\ Dr. Panjab Rao Deshmukh Krish Vidyapeeth, Akola - 444 104, Maharashtra, India \\ e-mail: jayalakshmimitnala@gmail.com
}

Received: 23 January 2014; Revised accepted: 13 March 2014

\section{A B S T R A C T}

The present investigation was carried at Nagarjun Medicinal and Aromatic Plants Garden. Dr. Panjabrao Deshmukh Krishi Vidyapeeth, Akola. There were four levels of nitrogen viz $0 \mathrm{~kg}\left(\mathrm{~N}_{0}\right), 40 \mathrm{~kg}\left(\mathrm{~N}_{1}\right), 60 \mathrm{~kg}\left(\mathrm{~N}_{2}\right), 80 \mathrm{~kg}\left(\mathrm{~N}_{3}\right)$ and three levels of phosphorus $0 \mathrm{~kg}\left(\mathrm{P}_{0}\right), 20 \mathrm{~kg}\left(\mathrm{P}_{1}\right), 40 \mathrm{~kg}\left(\mathrm{P}_{2}\right) \mathrm{ha}^{-1}$, tried in factorial randomized block design with three replications. The experimental findings revealed that growth parameters like plant height and number of tillers was recorded significantly highest due to the application of $80 \mathrm{~kg} \mathrm{~N} \mathrm{ha}^{-1}$ and $40 \mathrm{~kg} \mathrm{P}_{2} \mathrm{O}_{5} \mathrm{ha}^{-1}$. The fresh herbage yield was maximum with application of $80 \mathrm{~kg} \mathrm{~N}^{-1}$ and $40 \mathrm{~kg} \mathrm{P}_{2} \mathrm{O}_{5} \mathrm{ha}^{-1}$.

Key words: Cymbopogan martini, Nitrogen, Phosphorus, Herbage, Yield

The use of aromatic plants has been practiced since ancient times as is evidenced by records of Chinese, Egyptian, Mesopotamian Greek and Roman origin. Recent findings in Pakistan prove that it goes as for back as 5000 years. As a result of indiscriminate exploitation of this aromatic plant has almost disappeared from its natural habitat and at present the grass is being seen on the very lowermost slopes of some parts of satpuda hills. The time has therefore come to bring some of the aromatic plants under plough. Cymbopogan grasses are important for their essential oils twenty seven species are found in India. However, C. nardus, C. citratus, C. winterianus and $C$. motia found in cultivation only. Whereas, C. flexuosus, $C$. martinii and $C$. pendulus are found in both under cultivation and in wild state the rest of the species occur in wild state only (Gupta 1983). Cympogon martinii commonly known as Palmarosa or Rosha grass was selected for the study. It is a tall perennial tufted hedge native of most parts of sub tropical India. The species occurs in patches in open shrub forests in parts of Madhya Pradesh, Maharashtra and Andhra Pradesh where it is commercially collected and distilled for its oil. The total area under the crops ranges between 30,000 to 40,000 hectare. Palmarosa oil is very rich in geraniol (75$90 \%$ ) present both in free and bound form and oil is thus source of high grade geraniol for cosmetics and perfumery industry. Considering the importance of this crop every effort is made to increase the oil production of the crop by using improved nutrient management practices.

MATERIALS AND METHODS
A field experiment to study was conducted at Nagarjun Medicinal and Aromatic Plants Garden, Dr. Panjabrao Deshmukh Krishi Vidyapeeth Akola during Kharif 20072008. The soil site selected for the experiment was medium block and medium fertility status with good drainage. There were twelve treatment combinations, laid in factorial randomized block design with three replications. The treatment include four levels of nitrogen viz $0 \mathrm{~kg}\left(\mathrm{~N}_{0}\right), 40 \mathrm{~kg}$ $\left(\mathrm{N}_{1}\right), 60 \mathrm{~kg}\left(\mathrm{~N}_{2}\right), 80 \mathrm{~kg}\left(\mathrm{~N}_{3}\right)$ and three levels of phosphorus 0 $\mathrm{kg}\left(\mathrm{P}_{0}\right), 20 \mathrm{~kg}\left(\mathrm{P}_{1}\right), 40 \mathrm{~kg}\left(\mathrm{P}_{2}\right) \mathrm{ha}^{-1}$. The seed was mixed in $1: 6$ ratio of sand, and beaten to detach them from the accompanying glumes to ensure good germination. 4 weeks age seedlings were planted at the spacing of $45 \times 30 \mathrm{~cm}$. Protective irrigations were given at timely interval as and when required. Nitrogen was applied through urea in three split doses as per 2:1:1 basal dose, after $1^{\text {st }}$ cutting and after $2^{\text {nd }}$ cutting respectively. Phosphorus was applied as per the treatment as basal dose at the time of planting through single super phosphate. Potassium was applied as basal dose @ 40 $\mathrm{kg} \mathrm{ha}^{-1}$ through murate of potash to all the treatments. The first cutting was done after 110 days of plating $i \mathrm{e}$ at flowering stage, subsequently second and third cuttings were undertaken after 90 and 170 days after I cutting respectively. The second cutting was delayed or growth of the Palmarosa was adversely affected due to cold wave during December and January. The ten plants were selected randomly from each treatment per plot for recording observations. The observations on number of tillers at every harvesting were recorded. The plant height was recorded at every harvest and average height was calculated. Yields per plant were 
recorded from observational plants randomly under each treatment and averages were worked out per plant. The plant taken for weighed of fresh herbage was subjected to drying for the recording the dry weight of dry herbage and average were calculated per plant. On the basis of weight of fresh herbage per plant at each cutting was recorded and added to work out total fresh herbage yield per hectare in tones. Oil content in fresh herb was estimated at each cutting. The oil yield was calculated by multiplying herb yield with oil content.

\section{RESULTS AND DISCUSSION}

Effect of nitrogen on plant height

The results of the study revealed that nitrogen had significant effect on plant height at each cutting. Significantly highest plant height at $1^{\text {st }}, 2^{\text {nd }}, 3^{\text {rd }}$ cuttings (213.22, 210.25 and $227.79 \mathrm{~cm}$ respectively) was observed under the treatment $\mathrm{N}_{3}\left(80 \mathrm{~kg} \mathrm{~N} \mathrm{ha}^{-1}\right)$ followed by treatment $\mathrm{N}_{2}\left(60 \mathrm{~kg} \mathrm{~N} \mathrm{ha}{ }^{-1}\right)$. Maximum plant height attained by Palmarosa due to optimum proportion of nitrogen and carbohydrates compounds achieved with in the plants under treatment $80 \mathrm{~kg} \mathrm{~N} \mathrm{ha}^{-1}$ (Table 1). Significantly lowest plant height $\left(190.62,186.89\right.$ and $193.06 \mathrm{~cm}$ at $1^{\text {st }}, 2^{\text {nd }}$ and $3^{\text {rd }}$ cutting, respectively) was recorded under treatment $\mathrm{N}_{0}(0 \mathrm{~kg}$ $\mathrm{N} \mathrm{ha}^{-1}$ ). Low height due to disturbed relationship between fertilizer and carbohydrate compound. This data was in agreement with Maheswari et al. (1984) who also reported that plant height, fresh herbage yield were significantly increased over control up to application of $60 \mathrm{~kg} \mathrm{~N}+30 \mathrm{~kg}$ $\mathrm{P}+30 \mathrm{~kg} \mathrm{~K} /$ ha yield.

Table 1 Effect of nitrogen and phosphorus on plant height $(\mathrm{cm})$ at various cuttings

\begin{tabular}{|c|c|c|c|}
\hline \multirow{2}{*}{ Treatments } & \multicolumn{3}{|c|}{ Harvesting stages } \\
\hline & $1^{\text {st }}$ cutting & $2^{\text {nd }}$ cutting & $3^{\text {rd }}$ cutting \\
\hline \multicolumn{4}{|c|}{ Nitrogen levels $\left(\mathrm{kg} \mathrm{ha}^{-1}\right)$} \\
\hline $\mathrm{N}_{0}-0$ & 190.62 & 186.89 & 193.06 \\
\hline $\mathrm{N}_{1}-40$ & 191.40 & 187.16 & 193.35 \\
\hline $\mathrm{N}_{2}-60$ & 196.54 & 192.82 & 197.79 \\
\hline $\mathrm{N}_{3}-80$ & 213.22 & 210.25 & 222.79 \\
\hline 'F' test & Sig. & Sig. & Sig. \\
\hline $\mathrm{SE}(\mathrm{m}) \pm$ & 0.48 & 0.34 & 0.35 \\
\hline $\mathrm{CD}$ at $5 \%$ & 1.33 & 0.95 & 0.97 \\
\hline \multicolumn{4}{|c|}{ Phosphorus levels $\left(\mathrm{kg} \mathrm{ha}^{-1}\right)$} \\
\hline $\mathrm{P}_{0}-0$ & 193.02 & 189.36 & 196.98 \\
\hline$P_{1}-20$ & 198.05 & 194.78 & 201.22 \\
\hline$P_{2}-40$ & 203.31 & 199.50 & 207.05 \\
\hline 'F' test & Sig. & Sig. & Sig. \\
\hline $\mathrm{SE}(\mathrm{m}) \pm$ & 0.41 & 0.29 & 0.30 \\
\hline $\mathrm{CD}$ at $5 \%$ & 1.15 & 0.82 & 0.84 \\
\hline \multicolumn{4}{|c|}{ Interaction } \\
\hline 'F' test & Sig. & Sig. & Sig. \\
\hline $\mathrm{SE}(\mathrm{m}) \pm$ & 0.24 & 0.17 & 0.17 \\
\hline $\mathrm{CD}$ at $5 \%$ & 0.66 & 0.47 & 0.48 \\
\hline
\end{tabular}

\section{Effect of phosphorus on plant height}

Phosphorus application at various levels had significant effect on plant height at $1^{\text {st }}, 2^{\text {nd }}, 3^{\text {rd }}$ cuttings and significantly highest plant height $\left(203.31,199.50,207.05 \mathrm{~cm}\right.$ at $1^{\text {st }}, 2^{\text {nd }}$ and $3^{\text {rd }}$ cuttings respectively) was recorded, under treatment $\mathrm{P}_{2}\left(40 \mathrm{~kg} \mathrm{P}_{2} \mathrm{O}_{5} \mathrm{ha}^{-1}\right)$ followed by $\mathrm{P}_{1}$. Phosphorus is an important growth promoting element and therefore, increases in growth as a result of application of phosphorus. The data was in agreement with Singh et al. (1991). They observed highest plant height, and tiller production at each cutting with the application of P levels upto $35 \mathrm{~kg} \mathrm{ha}^{-1}$.

\section{Interaction effect on plant height}

Interaction effect of nitrogen and phosphorus on plant height was found significant at each cutting.

Table 1a Interaction effect of nitrogen and phosphorus on plant height $(\mathrm{cm})$ at $1^{\text {st }}$ cutting

\begin{tabular}{|c|c|c|c|c|}
\hline \multirow{2}{*}{ Treatments } & \multicolumn{4}{|c|}{ Phosphorus levels kg ha ${ }^{-1}$} \\
\hline & $\mathrm{P}_{0}$ & $\mathrm{P}_{1}$ & $\mathrm{P}_{2}$ & Mean $(\mathrm{N})$ \\
\hline \multicolumn{5}{|c|}{ Nitrogen levels $\left(\mathrm{kg} \mathrm{ha}^{-1}\right)$} \\
\hline $\mathrm{N}_{0}$ & 189.46 & 190.90 & 191.50 & 190.62 \\
\hline $\mathrm{N}_{1}$ & 187.83 & 192.66 & 193.76 & 191.4 \\
\hline $\mathrm{N}_{2}$ & 193.96 & 196.66 & 199.00 & 196.54 \\
\hline $\mathrm{N}_{3}$ & 201.00 & 209.66 & 229.00 & 213.22 \\
\hline Mean $(\mathrm{P})$ & 193.02 & 198.05 & 203.31 & \\
\hline 'F' test & Sig. & & & \\
\hline $\mathrm{SE}(\mathrm{m}) \pm$ & 0.24 & & & \\
\hline $\mathrm{CD}$ at $5 \%$ & 0.66 & & & \\
\hline
\end{tabular}

Table $1 \mathrm{~b}$ Interaction effect of nitrogen and phosphorus on plant height $(\mathrm{cm})$ at $2^{\text {nd }}$ cutting

\begin{tabular}{|c|c|c|c|c|}
\hline \multirow{2}{*}{ Treatments } & \multicolumn{4}{|c|}{ Phosphorus levels $\mathrm{kg} \mathrm{ha}^{-1}$} \\
\hline & $\mathrm{P}_{0}$ & $\mathrm{P}_{1}$ & $\mathrm{P}_{2}$ & $\operatorname{Mean}(\mathrm{N})$ \\
\hline \multicolumn{5}{|c|}{ Nitrogen levels $\left(\mathrm{kg} \mathrm{ha}^{-1}\right)$} \\
\hline $\mathrm{N}_{0}$ & 179.33 & 190.00 & 191.36 & 186.89 \\
\hline $\mathrm{N}_{1}$ & 185.66 & 186.83 & 189.00 & 187.16 \\
\hline $\mathrm{N}_{2}$ & 190.16 & 193.33 & 195.00 & 192.82 \\
\hline $\mathrm{N}_{3}$ & 199.13 & 208.96 & 222.66 & 210.25 \\
\hline Mean (P) & 189.36 & 194.78 & 199.50 & - \\
\hline 'F' test & Sig. & & & \\
\hline $\mathrm{SE}(\mathrm{m}) \pm$ & 0.17 & & & \\
\hline $\mathrm{CD}$ at $5 \%$ & 0.47 & & & \\
\hline
\end{tabular}

Table 1c Interaction effect of nitrogen and phosphorus on plant height $(\mathrm{cm})$ at $3^{\text {rd }}$ cutting

\begin{tabular}{lcccc}
\hline \multirow{2}{*}{ Treatments } & \multicolumn{4}{c}{ Phosphorus levels kg ha } \\
\cline { 2 - 5 } & $\mathrm{P}_{0}$ & $\mathrm{P}_{1}$ & $\mathrm{P}_{2}$ & Mean (N) \\
\hline \multicolumn{4}{c}{ Nitrogen levels $\left(\mathrm{kg} \mathrm{ha}^{-1}\right)$} \\
$\mathrm{N}_{0}$ & 190.83 & 193.16 & 195.20 & 193.06 \\
$\mathrm{~N}_{1}$ & 192.36 & 194.43 & 193.26 & 193.35 \\
$\mathrm{~N}_{2}$ & 195.13 & 198.76 & 199.50 & 197.79 \\
$\mathrm{~N}_{3}$ & 209.60 & 218.53 & 240.26 & 222.79 \\
Mean (P) & 196.98 & 201.22 & 207.05 & - \\
$\mathrm{F}^{\prime}$ test & $\mathrm{Sig}$. & & & \\
$\mathrm{SE}(\mathrm{m}) \pm$ & 0.17 & & & \\
$\mathrm{CD}$ at 5\% & 0.45 & & & \\
\hline
\end{tabular}

It is evident from the data (Table 2a,b,c) that the significantly highest plant height at each cutting was recorded with the treatment combination of $\mathrm{N}_{3}+\mathrm{P}_{2}$ i e $80 \mathrm{~kg}$ 
$\mathrm{N}+40 \mathrm{~kg} \mathrm{P}_{2} \mathrm{O}_{5}$ ha $^{-1}$ followed by the treatment combination of $\mathrm{N}_{3+} \mathrm{P}_{1}$ i e $80 \mathrm{~kg} \mathrm{~N}+20 \mathrm{~kg} \mathrm{P}_{2} \mathrm{O}_{5} \mathrm{ha}^{-1}$.

Table 2 Effect of nitrogen and phosphorus on number of tillers at various cuttings

\begin{tabular}{|c|c|c|c|}
\hline \multirow{2}{*}{ Treatments } & \multicolumn{3}{|c|}{ Harvesting stages } \\
\hline & $1^{\text {st }}$ cutting & $2^{\text {nd }}$ cutting & $3^{\text {rd }}$ cutting \\
\hline \multicolumn{4}{|c|}{ Nitrogen levels $\left(\mathrm{kg} \mathrm{ha}^{-1}\right)$} \\
\hline $\mathrm{N}_{0}-0$ & 13.30 & 14.11 & 20.30 \\
\hline $\mathrm{N}_{1}-40$ & 19.10 & 21.00 & 22.00 \\
\hline $\mathrm{N}_{2}-60$ & 23.00 & 24.11 & 25.33 \\
\hline $\mathrm{N}_{3}-80$ & 28.00 & 28.11 & 28.44 \\
\hline 'F' test & Sig. & Sig. & Sig. \\
\hline $\mathrm{SE}(\mathrm{m}) \pm$ & 0.55 & 0.52 & 0.44 \\
\hline $\mathrm{CD}$ at $5 \%$ & 1.53 & 1.46 & 1.23 \\
\hline \multicolumn{4}{|c|}{ Phosphorus levels $\left(\mathrm{kg} \mathrm{ha}^{-1}\right)$} \\
\hline$P_{0}-0$ & 19.40 & 20.50 & 22.33 \\
\hline $\mathrm{P}_{1}-20$ & 20.80 & 21.91 & 24.25 \\
\hline$P_{2}-40$ & 22.32 & 23.08 & 25.49 \\
\hline 'F' test & Sig. & Sig. & Sig. \\
\hline $\mathrm{SE}(\mathrm{m}) \pm$ & 0.47 & 0.45 & 0.38 \\
\hline $\mathrm{CD}$ at $5 \%$ & 1.32 & 1.26 & 1.06 \\
\hline \multicolumn{4}{|c|}{ Interaction } \\
\hline 'F' test & NS & Sig. & NS \\
\hline $\mathrm{SE}(\mathrm{m}) \pm$ & 0.27 & 0.26 & 0.22 \\
\hline $\mathrm{CD}$ at $5 \%$ & - & 0.73 & - \\
\hline
\end{tabular}

Effect of nitrogen on number of tillers

The data further indicating that significantly highest number of tillers at $1^{\text {st }}, 2^{\text {nd }}$ and $3^{\text {rd }}$ cutting $(28,28.1,28.44$ respectively) was observed under treatment $\mathrm{N}_{3}\left(80 \mathrm{~kg} \mathrm{~N}^{-}\right.$ $\left.{ }^{1}\right)$ followed by treatment $\mathrm{N}_{2}\left(60 \mathrm{~kg} \mathrm{~N} \mathrm{ha}^{-1}\right)$. Significantly lowest number of tillers $(13.30,14.11,20.30)$ at $1^{\text {st }}, 2^{\text {nd }}$ and $3^{\text {rd }}$ cuttings respectively were recorded under treatment $\mathrm{N}_{0}$ $\left(0 \mathrm{~kg} \mathrm{~N} \mathrm{ha}{ }^{-1}\right)$. Formation of plant parts above the soil represent photosynthesis apparatus in conversion of solar energy into chemical energy totally depends on the utilization of carbohydrates and other metabolities in roots accelerated by nitrogen uptake which ultimately gives more number of tillers (Table 2). This results are supported by Bommegowda et al. (1980a) who also revealed that application of 75 to $100 \mathrm{~kg} \mathrm{~N} \mathrm{ha}^{-1}$ significantly increase tiller number and leaf area.

\section{Effect of phosphorus}

Favourable effect of various phosphorus levels was noticed on the number of tillers per plant at each cutting. Significantly highest number of tillers at $1^{\text {st }}, 2^{\text {nd }}$ and $3^{\text {rd }}$ cuttings $(22.32,23.08,25.49$, respectively) was observed under treatment $\mathrm{N}_{3}\left(80 \mathrm{~kg} \mathrm{~N}^{-1}\right)$ followed by treatment $\mathrm{N}_{2}$ $\left(60 \mathrm{~kg} \mathrm{~N} \mathrm{ha}^{-1}\right)$. The lowest number of tillers $(19.40,20.50$, 22.33) was recorded under treatment $\mathrm{P}_{0}\left(0 \mathrm{~kg} \mathrm{P}_{2} \mathrm{O}_{5} \mathrm{ha}^{-1}\right)$. Phosphorus having close relationship in cell division and development. It stimulates early root development and growth. So, it might be the reason to improve tiller number due to increased application of phosphorus. This data also supported by the findings of Singh et al. (1991).
Interaction effect of nitrogen and phosphorus on number of tillers

Interaction effect of nitrogen and phosphorus on number of tillers was found significant at $2^{\text {nd }}$ cutting, however in $1^{\text {st }}$ and $2^{\text {nd }}$ cutting it was found non significant.

Table 2a Interaction effect of nitrogen and phosphorus on number of tillers at $2^{\text {nd }}$ cutting

\begin{tabular}{lcccc}
\hline \multirow{2}{*}{ Treatments } & \multicolumn{4}{c}{ Phosphorus levels kg ha } \\
\cline { 2 - 5 } & $\mathrm{P}_{0}$ & $\mathrm{P}_{1}$ & $\mathrm{P}_{2}$ & Mean (N) \\
\hline \multirow{4}{*}{ Nitrogen levels $\left(\mathrm{kg} \mathrm{ha}^{-1}\right)$} \\
$\mathrm{N}_{0}$ & 13.00 & 14.00 & 15.33 & 14.11 \\
$\mathrm{~N}_{1}$ & 20.00 & 21.00 & 22.00 & 21.00 \\
$\mathrm{~N}_{2}$ & 23.00 & 24.33 & 25.00 & 24.11 \\
$\mathrm{~N}_{3}$ & 26.00 & 28.33 & 30.00 & 28.11 \\
Mean $(\mathrm{P})$ & 20.50 & 21.91 & 23.08 & - \\
'F' test & Sig. & & & \\
$\mathrm{SE}(\mathrm{m}) \pm$ & 0.26 & & & \\
$\mathrm{CD}$ at $5 \%$ & 0.73 & & & \\
\hline
\end{tabular}

The data revealed that significantly highest number of tillers was recorded at the treatment combination $\mathrm{N}_{3}+\mathrm{P}_{2}(80$ $\mathrm{kg} \mathrm{N}+40 \mathrm{~kg} \mathrm{P}_{2} \mathrm{O}_{5} \mathrm{ha}^{-1}$ ) followed by treatment combination $\mathrm{N}_{3}+\mathrm{P}_{1}\left(80 \mathrm{~kg} \mathrm{~N}+20 \mathrm{~kg} \mathrm{P}_{2} \mathrm{O}_{5} \mathrm{ha}^{-1}\right)$. Nitrogen phosphorus interaction had significant effect on growth (plant height, number of tillers) revealed that the beneficial effect of nitrogen on vegetative growth was increased by higher level of phosphorus fertilization and vice-versa. It thus appears that the nitrogen and phosphorus on growth of Palmarosa is complimentary to each other.

Table 3 Effect of nitrogen and phosphorus on fresh herbage yield (tonne ha' ${ }^{-1}$ )

\begin{tabular}{|c|c|c|c|c|}
\hline \multirow{2}{*}{ Treatments } & \multicolumn{4}{|c|}{ Harvesting stages } \\
\hline & $1^{\text {st }}$ cuttin & $2^{\text {nd }}$ cuttir & cuttins & Total yield \\
\hline \multicolumn{5}{|c|}{ Nitrogen levels $\left(\mathrm{kg} \mathrm{ha}^{-1}\right)$} \\
\hline $\mathrm{N}_{0}$ & 3.52 & 3.84 & 4.13 & 11.49 \\
\hline $\mathrm{N}_{1}$ & 4.16 & 4.81 & 5.03 & 14.00 \\
\hline $\mathrm{N}_{2}$ & 4.65 & 5.38 & 5.66 & 15.69 \\
\hline $\mathrm{N}_{3}$ & 5.21 & 5.92 & 6.13 & 17.26 \\
\hline 'F' test & Sig. & Sig. & Sig. & Sig. \\
\hline $\mathrm{SE}(\mathrm{m}) \pm$ & 0.08 & 0.05 & 0.06 & 0.56 \\
\hline $\mathrm{CD}$ at $5 \%$ & 0.24 & 0.16 & 0.19 & 1.52 \\
\hline \multicolumn{5}{|c|}{ Phosphorus levels $\left(\mathrm{kg} \mathrm{ha}^{-1}\right)$} \\
\hline $\mathrm{P}_{0}$ & 4.39 & 4.70 & 5.00 & 14.09 \\
\hline $\mathrm{P}_{1}$ & 4.43 & 5.05 & 5.30 & 14.78 \\
\hline $\mathrm{P}_{2}$ & 4.53 & 5.17 & 5.40 & 15.1 \\
\hline 'F' test & Sig. & Sig. & Sig. & Sig. \\
\hline $\mathrm{SE}(\mathrm{m}) \pm$ & 0.07 & 0.05 & 0.05 & 0.22 \\
\hline $\mathrm{CD}$ at $5 \%$ & 0.21 & 0.13 & 0.16 & 0.70 \\
\hline \multicolumn{5}{|c|}{ Interaction } \\
\hline 'F' test & NS & NS & NS & NS \\
\hline $\begin{array}{l}\mathrm{SE}(\mathrm{m}) \pm \\
\mathrm{CD} \text { at } 5 \%\end{array}$ & 0.04 & 0.02 & 0.03 & 0.05 \\
\hline
\end{tabular}

Effect of nitrogen on fresh herbage yield

Nitrogen had significant effect on fresh herbage yield at each cutting under study. Significantly highest fresh herbage 
yield at $1^{\text {st }}, 2^{\text {nd }}, 3^{\text {rd }}$ cuttings and total yield $(5.21,5.92,6.13$, $17.26 \mathrm{t} \mathrm{ha}^{-1}$ respectively) was observed under treatment $\mathrm{N}_{3}$ $\left(80 \mathrm{~kg} \mathrm{~N} \mathrm{ha}^{-1}\right)$ followed by treatment $\mathrm{N}_{2}\left(60 \mathrm{~kg} \mathrm{~N} \mathrm{ha}^{-1}\right)$.

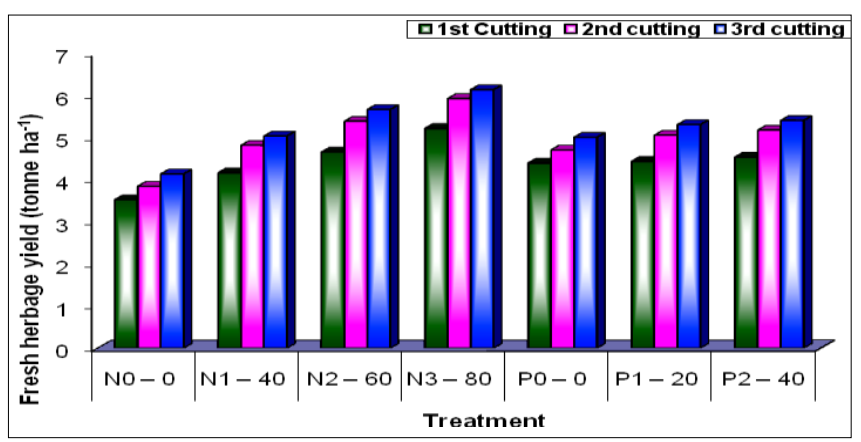

Fig 1 Effect of nitrogen and phosphorus on fresh herbage yield at various cuttings

Whereas, lowest fresh herbage yield $(3.52,3.84,4.13$, $11.49 \mathrm{t} \mathrm{ha}^{-1}$ ) respectively was recorded under treatment $\mathrm{N}_{0}$ $\left(0 \mathrm{~kg} \mathrm{~N} \mathrm{ha}{ }^{-1}\right)$. In general each level of nitrogen was significantly superior to the preceding level. This is to be expected as nitrogen being a known growth promoting factor its application would naturally increase the vegetative growth and hence fresh herbage yield. These results were in agreement with findings of Singh and Singh (1992) who have also reported increased fresh herbage and oil yield with the application of $150 \mathrm{~kg} \mathrm{~N} \mathrm{ha}^{-1}$.
Effect of phosphorus on fresh herbage yield

Phosphorus application had significant effect on fresh herbage yield at each cutting. Significantly highest fresh herbage yield $\left(4.53,5.17,5.4,15.1 \mathrm{t} \mathrm{ha}^{-1}\right.$, respectively) was recorded at $1^{\text {st }}, 2^{\text {nd }}$ and $3^{\text {rd }}$ cuttings and total yield under treatment $\mathrm{P}_{2}\left(40 \mathrm{~kg} \mathrm{P}_{2} \mathrm{O}_{5} \mathrm{ha}^{-1}\right)$. However, treatment $\mathrm{P}_{1}(20$ kg $\mathrm{P}_{2} \mathrm{O}_{5}$ ha $\left.^{-1}\right)$ was statistically at par with treatment $\mathrm{P}_{2}(40$ $\mathrm{kg} \mathrm{P}_{2} \mathrm{O}_{5} \mathrm{ha}^{-1}$ ) at all three cuttings, as phosphorus stimulates vegetative growth and flowering. So, it might be the reason for increased fresh herbage yield due to phosphorus application.

\section{Interaction effect on fresh herbage yield}

The interaction effect between nitrogen and phosphorus on fresh herbage yield was found non significant. However, in depth study it may be observed that with higher dose of phosphorus, beneficial effect of nitrogen on fresh herbage yield was fairly enhanced. Similarly with the higher dose of nitrogen, phosphorus increased the herbage yield to a greater extent than with lower doses of nitrogen. Therefore, role of nitrogen and phosphorus both themselves inherently positive growth promoting factors, are complimentary to each other.

Growth parameters like plant height and number of tillers were recorded significantly highest due to the application of $80 \mathrm{~kg} \mathrm{~N} \mathrm{ha}^{-1}$ and $40 \mathrm{~kg} \mathrm{P}_{2} \mathrm{O}_{5} \mathrm{ha}^{-1}$. The fresh herbage yield was maximum with application of $80 \mathrm{~kg} \mathrm{~N}$ per hectare and $40 \mathrm{~kg} \mathrm{P}_{2} \mathrm{O}_{5} \mathrm{ha}^{-1}$.

\section{LITERATURE CITED}

Bommegowada A, Narayana M R, Krishnamurthy K and Chandrasekhar G. 1980a. Economics of Java citronella (Cymbopogon winterianus Jowitt). Mysore Journal of Agricultural Sciences 14: 480-482.

Bommegowada A, Joshi S, Narayana M R and Krishnamurthy K. 1980b. Effect of nitrogen nutrition on oil per cent and oil glands in Java citronella (Cymbopogon winterianus Jowitt). Mysore Journal of Agricultural Sciences 14: 477-479.

Gupta B K. 1983. Indian Cymbopogans, their distribution and existence. Indian Perfumer 27(2): 108-111.

Maheswari S K, Yadav S and Gupta R S. 1984. Fertilizer needs of Palmarosa oil grass (Cymbopogon martinii Var. Motia) under rainfed condition of Madhya Pradesh. Indian Perfumer 28(2): 77-81.

Singh K and Singh D V. 1992. Effect of rates and sources of nitrogen application on yield and nutrient uptake of citronella java (Cymbopogon winterianus Jowitt). Fertilizer Research 33(3): 187-191.

Singh K, Chowdary A, Subrahmanyam K, Chatterjee B N and Singh D V. 1991. Growth and yield response of citronella Java (Cymbopogon winterianus Jowitt) to phosphorus and potassium levels at high nitrogen. Journal of Potassium Research 7(1): 35-46. 